\section{Vulnerabilidades e situações de riscos relacionados ao uso de agrotóxicos na fruticultura irrigada}

\section{Vulnerability and risk situations related to the use of pesticides in irrigated fruit farming}

\author{
Cheila Nataly Galindo Bedor ${ }^{1}$ \\ Lara Oliveira Ramos \\ Paulo José Pereira ${ }^{3}$ \\ Marco Antônio Vasconcelos Rêgo ${ }^{4}$ \\ Antonio Carlos Pavão 5

\section{Lia Giraldo da Silva Augusto ${ }^{6}$} \\ ${ }^{1}$ Colegiado de Medicina da Universidade Federal do Vale do São Francisco \\ ${ }^{2}$ Curso de Enfermagem da Universidade Federal do Vale do São Francisco \\ ${ }^{3}$ Colegiado de Engenharia de Produção da Universidade Federal do Vale do São \\ Francisco \\ ${ }^{4}$ Departamento de Medicina Preventiva e Social da Universidade Federal da \\ Bahia \\ ${ }^{5}$ Departamento de Química Fundamental da Universidade Federal de \\ Pernambuco \\ ${ }^{6}$ Departamento de Saúde Coletiva do Centro de Pesquisa Aggeu Magalhães da \\ FIOCRUZ
}

Financiamento: Conselho Nacional de Desenvolvimento Científico e Tecnológico - № do processo $409837 / 2006-7$

Correspondência: Cheila Nataly Galindo Bedor. Colegiado de Medicina da Universidade Federal do Vale do São Francisco. Av. José de Sá Maniçoba, s/n, Centro - CEP 56.304-205 Petrolina, PE. Email: cheila.bedor@univasf.edu.br

\section{Resumo}

A determinação de situações de riscos na população que faz uso de agrotóxico é complexa. O objetivo desse estudo foi conhecer o contexto social, econômico e cultural e algumas das vulnerabilidades para a saúde relacionadas com o processo produtivo químico dependente da fruticultura do Vale do São Francisco. Questionário semiestruturado foi aplicado a uma amostra aleatória de trabalhadores rurais, para obtenção de informações socioeconômicas e culturais, assim como a morbidade relacionada com intoxicação por agrotóxicos. Os trabalhadores rurais da região têm em sua maioria baixo grau de escolaridade, sendo esta uma importante vulnerabilidade para compreensão da rotulagem dos agrotóxicos e sua implicação toxicológica e ambiental. Foi observado uso indiscriminado de agrotóxicos em condições inseguras de trabalho que comprometem a saúde dos expostos. A sintomatologia sugestiva de intoxicação por agrotóxicos aponta para quadros relacionados com exposição à organofosforados, carbamatos e piretróides, em congruência com os biocidas utilizados na fruticultura. $7 \%$ da população estudada referiram ter sofrido pelo menos um caso de intoxicação no decorrer da vida. A assistência técnica relacionada com o manejo de agrotóxicos é precária e não se observaram ações de proteção no âmbito da saúde, do trabalho, da previdência ou do ambiente. Estas vulnerabilidades institucionais corroboram aquelas relacionadas com o modelo tecnológico que objetiva meramente a produtividade $\mathrm{e}$ o rendimento financeiro.

Palavras-chave: Agrotóxico. Vulnerabilidade. Situações de risco. Saúde do trabalhador. Saúde ambiental. Zona semi-árida. 


\section{Abstract}

Determining the risks in a population that uses pesticides is a complex task. The purpose of this study was to outline the socioeconomic and cultural context and some of the health vulnerabilities related to fruit production in the São Francisco River valley in Brazil. Semi-structured questionnaires were applied to random samples of farm workers to obtain data on social, economic and cultural characteristics, as well as the morbidity related to pesticide poisoning. These farm workers have low level of schooling, and this is a major vulnerability for understanding the labels of pesticides and their toxicological and environmental implications. This study found indiscriminate use of pesticides in unsafe and unhealthy conditions of work that compromise the health of exposed workers. Symptoms suggestive of pesticide poisoning are closely related to exposure to organophosphates, carbamates and pyrethroid, which match pesticides used in fruit farming. Seven percent of the population reported poisoning events at some point in their lives. Technical assistance related to the management of pesticides is poor, and actions toward the improvement of health, environment and work conditions were not seen. These vulnerabilities corroborate those related to a technological model that aims merely at productivity and good financial performance.

Keywords: Pesticides. Vulnerability. Risk situations. Occupational health. Environmental health. Semi-arid region.

\section{Introdução}

A ampla utilização de agrotóxicos no sistema produtivo rural é um grave problema para a saúde e para o ambiente, por contaminação do solo, da água e do $\operatorname{ar}^{1}$. Estima-se que ocorram no mundo cerca de três milhões de intoxicações agudas por agrotóxico com 220 mil mortes, sendo $70 \%$ dessas provenientes dos países em desenvolvimento ${ }^{2}$. Os trabalhadores rurais constituem um grupo populacional reconhecidamente vulnerável aos efeitos danosos dos agrotóxicos na saúde ${ }^{3}$. Relatos da literatura indicam que a cada caso de intoxicação aguda há uma expectativa de oito casos de intoxicação crônica ${ }^{4}$. Segundo Augusto et al. ${ }^{5}$, mesmo sendo o Brasil um dos principais países consumidores de agrotóxicos na América Latina, ainda se observa grande escassez de informações sobre os efeitos decorrentes da exposição humana a esses compostos.

Os agrotóxicos representam um grupo heterogêneo de compostos com variadas estruturas químicas e com diferentes toxicicidades $^{6}$. Por conseguinte, monitorar e controlar seu uso continua sendo um desafio para a vigilância da saúde e ambiental.

A baixa percepção das situações de riscos a que está exposto o indivíduo e também o seu coletivo é uma questão presente no conjunto de vulnerabilidades existentes no âmbito do uso dessa tecnologia para diversos fins ${ }^{1}$. Reconhecer os condicionantes sociais, culturais e econômicos presentes no processo produtivo agrário químico-dependente é uma necessidade para minimizar os danos à saúde e ao ambiente decorrente do uso de agrotóxicos ${ }^{7}$. Diversos estudos referem que esses condicionantes contextuais também determinam o nível de percepção social dessas situações de riscos (Popper et al. ${ }^{8}$, na Guatemala; Mwanthi et al. ${ }^{9}$, no Quênia; McDougall et al. ${ }^{10}$; na Ilha de Santa Lúcia no Caribe; e Stewart ${ }^{11}$, no Egito).

No Brasil, a região do submédio do Vale do São Francisco é um dos principais polos produtores e exportadores de frutas, sendo especializada na produção de uva e manga. 
A irrigação associada ao uso de agrotóxicos e fertilizantes é o modelo utilizado para a produção dessas monoculturas.

O objetivo desse estudo foi conhecer o contexto social, econômico e cultural e algumas das vulnerabilidades para a saúde relacionadas com o processo produtivo químico-dependente da fruticultura do Vale do São Francisco. Busca-se contribuir para ações de proteção da saúde dos trabalhadores rurais frente ao uso de agrotóxicos em um modelo produtivo agro-exportador.

\section{Método}

Trata-se de um estudo descritivo, realizado no período de 2006 a 2007, no polo fruticultor do submédio do Vale de São Francisco. Especificamente nos projetos de irrigação Nilo Coelho, Maria Tereza e Bebedouro, localizados em Petrolina - PE, e os de Curaçá, Maniçoba e Tourão, situados em Juazeiro - BA e cidades circunvizinhas. O mapa da região do submédio do vale do São Francisco esta identificado na Figura 1.
Como estratégia de seleção da amostra, as áreas foram escolhidas por estratificação segundo o tamanho dos lotes. Procurouse garantir a representatividade de todos os tipos de propriedades que, segundo a Companhia do Vale do São Frâncico (CODEVASF), correspondem a cerca de 3.040, dividas em 2.874 pequenas propriedades (lotes com até 12 hectares), 133 pequenas e médias empresas (12 a 50 hectares) e 33 grandes empresas (áreas com mais de 50 hectares).

População de estudo: amostra de 300 indivíduos, randomizada com $95 \%$ de confiança e um erro de 2,5\%. Dada à dificuldade de se saber a priori a quantidade de trabalhadores rurais em todos os lotes, foi utilizada para a base de cálculo do tamanho da amostra a prevalência de $5 \%$ de referência de intoxicação por agrotóxico encontrada na região por Ramos et al. ${ }^{12}$, no Projeto de Irrigação Nilo Coelho.

A amostra foi composta de indivíduos que utilizam ou já utilizaram agrotóxicos e que se encontravam trabalhando nas

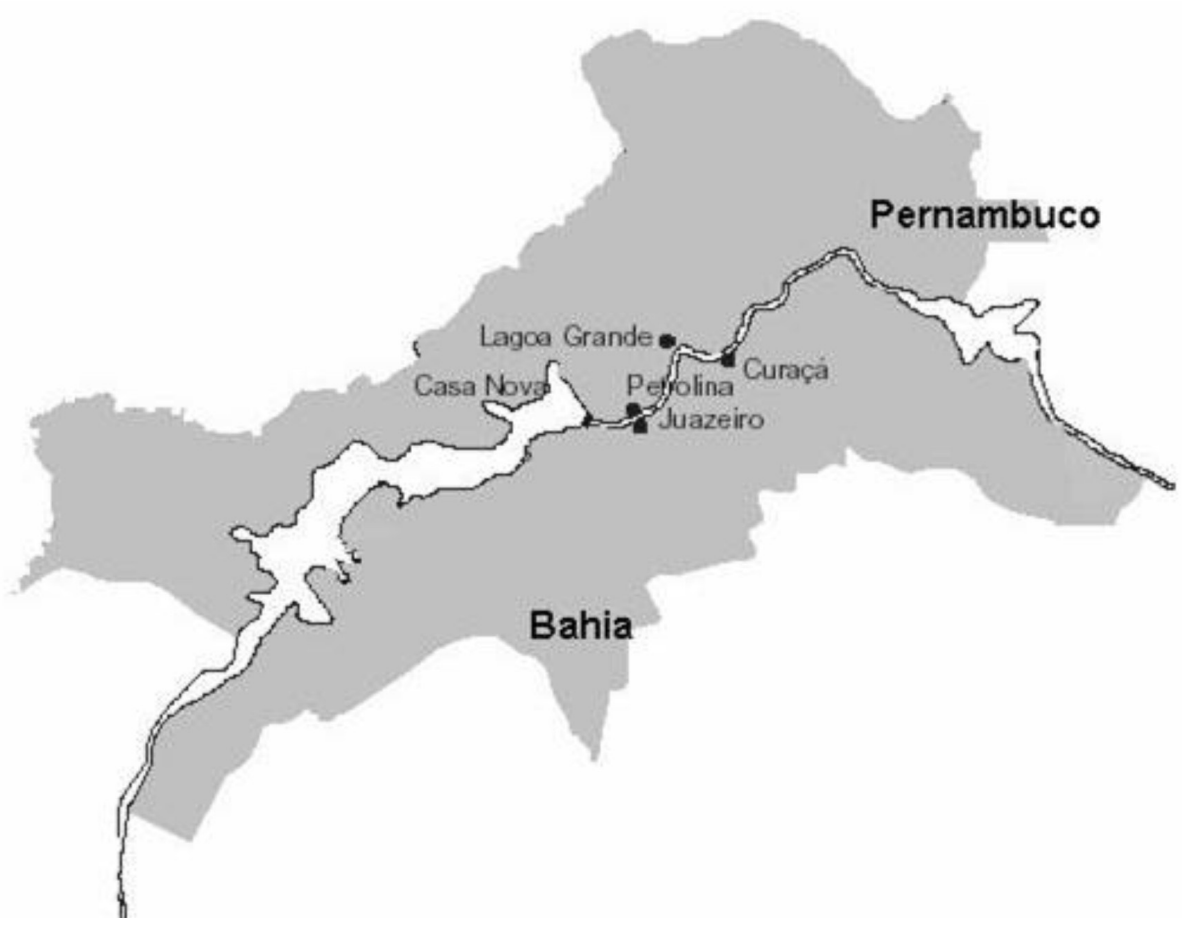

Figura 1 - Mapa do Vale do São Francisco com destaque dos municípios onde foram coletados os dados.

Figure 1 - Map of the São Francisco Valleywith the municipalities where data were collected. 
lavouras no momento das visitas. Foram entrevistados cerca de 1 trabalhador por pequena propriedade, 1 por pequena ou média empresa e cerca de 10 trabalhadores em cada grande empresa visitada. Como critério de exclusão foram eliminados da amostra 17 indivíduos que tiveram dificuldade de informação, o que não interferiu no perfil da amostra, totalizando 283 indivíduos, sendo 146 trabalhadores de pequenas propriedades; 73 de médio porte e 64 de grande porte. As propriedades foram escolhidas aleatoriamente através de sorteio.

Foi utilizado um questionário semiestruturado para a coleta de dados primários sobre condições de vida, ambiente e trabalho e de morbidade referida. Para avaliação da exposição ocupacional aos agrotóxicos, foram coletadas informações sobre o tipo e a maneira de utilização desses produtos, o intervalo de aplicação dos mesmos, se os indivíduos receberam orientação técnica sobre a utilização dos agrotóxicos, se utilizavam receituário para sua compra e se liam a bula antes de sua utilização. Além disso, foi verificado quais dos Equipamentos de Proteção individual (EPI), preconizados para proteção química, eram utilizados por esses trabalhadores rurais na hora em que estava sendo aplicado agrotóxico. A variável jornada de trabalho foi referente à quantidade de horas que o trabalhador permanecia nas lavouras durante o dia.

O dado de intoxicação por agrotóxico foi obtido através dos trabalhadores rurais que afirmaram já ter sofrido, ao longo da vida, intoxicação por agrotóxico confirmada por atendimento médico. Já os sintomas listados são aqueles referidos por esses trabalhadores após ou durante a aplicação de agrotóxico.

Os dados secundários, como os registros de intoxicação por agrotóxicos do município de Petrolina e os dados da Previdência Social, foram utilizados para caracterização de contexto das condições sociais, econômicas e culturais da região, cujas informações foram obtidas de documentos oficiais e publicações.

As classificações dos agrotóxicos, segun- do classe, ingrediente ativo, grupo químico, classificação toxicológica e ambiental foram obtidas mediante consulta ao Sistema de Agrotóxicos Fitossanitários (AGROFIT) do Ministério de Agricultura, Pecuária e Abastecimento e o Sistema de Informação sobre Agrotóxicos (SIA) da Agência Nacional de Vigilância Sanitária, no período de novembro de 2006 a agosto de 2007.

O presente estudo teve aprovação do comitê de ética do Centro de Pesquisa Aggeu Magalhães - FIOCRUZ (CAEE: 0033.0.095.000-06).

\section{Resultados}

Os resultados desse estudo são baseados nos questionários semiestruturados respondidos pelos produtores rurais da região estudada. Três grandes empresas não permitiram entrevistas com seus trabalhadores, sendo essas substituídas por outras propriedades de área equivalente.

A Tabela 1 mostra as características sociodemográficas dos indivíduos estudados. Destaca-se que $69 \%$ eram empregados e $31 \%$ eram proprietários rurais que trabalhavam diretamente na sua terra, pequenas propriedades ou pequenas e médias empresas; a maioria era do sexo masculino, de idade entre 15 e 79 anos, com média de 35 anos $( \pm 11,8)$. A escolaridade é baixa, com $12 \%$ de analfabetos e $64 \%$ apenas com o primeiro grau. A renda da maioria (55\%) é igual ou menor a 1 salário mínimo.

A monocultura é predominante na região, sendo a manga e a uva as principais culturas, cultivadas respectivamente por $65 \%$ e $49 \%$ dos agricultores entrevistados. Foram citados cerca de 50 nomes comerciais de agrotóxicos, com predomínio do Folisuper (parationa metílica), Vertimec (abamectina) e Dormex (cianamida).

Os organofosforados foram os mais citados entre as classes de agrotóxicos. A frequência de aplicação desses compostos nas plantações varia para cada cultura, mas em média é de quatro vezes por mês. Nenhum agricultor citou fazer uso de agrotóxicos proibidos. A Tabela 2 mostra características 
Tabela 1 - Características sociodemográficas dos agricultores na fruticultura do submédio do Vale do São Francisco

Table 1 - Socio-demographic characteristics of farmers in the fruit farming of the San Francisco Valley

\begin{tabular}{llcc}
\hline Características & & $\mathrm{N}=283$ & $(\%)$ \\
\hline Sexo & Masculino & 234 & 83 \\
Escolaridade & Feminino & 49 & 17 \\
& Analfabetos & 34 & 12 \\
& $1^{\circ}$ grau completo & 181 & 64 \\
& $2^{\circ}$ grau completo & 57 & 20 \\
Relação de trabalho & 11 & 4 \\
& 3० grau ou mais & 155 & 55 \\
& Carteira assinada & 31 & 11 \\
& Não possuem carteira assinada & 5 & 2 \\
Jornada de trabalho & Contratados por diária & 15 & 1 \\
& Contratado em época de safra & 234 & 5 \\
& 6 a 7 horas/dia & 15 & 53 \\
& 8 a 9 horas/dia & 11 & 4 \\
& 10 a 11 horas/dia & 8 & 3 \\
\hline
\end{tabular}

dos principais agrotóxicos utilizados e de seus manuseios.

Entre as embalagens vazias de agrotóxicos, $7 \%$ são queimadas; $13 \%$ ficam retidas nas propriedades, $2 \%$ são devolvidas à loja onde foram compradas e $78 \%$ são entregues à Associação do Comércio Agropecuário do Vale do São Francisco (ACAVASF). Porém, segundo os trabalhadores rurais. a ACAVASF há cerca de um ano não realiza a coleta na região, o que justifica a observação de várias embalagens de agrotóxicos jogadas no meio do mato dos projetos visitados.

Quanto à exposição individual a agrotóxicos, $62 \%$ dos entrevistados manipulam esses produtos há cerca de 10 a 30 anos, $4 \%$ há menos de um ano, e 34\% não responderam a essa questão.

O EPI completo é utilizado por $72 \%$ da população estudada, sendo observado principalmente o uso de máscara e luva naqueles que os utilizam de maneira incompleta (Tabela 2). Os produtores que responderam não utilizar EPI estão entre os pequenos produtores e trabalhadores de pequenas e médias empresas.

Cerca de $30 \%$ dos agricultores, a maioria do sexo masculino (85\%), queixaram-se de pelo menos um sintoma após a aplicação ou de estar dentro da área onde foi aplicado agrotóxico. A média desses sintomas foi de 1 (29\%) entre os agricultores que não sofreram intoxicação e de 2 a 3 (25\% e 20\%, respectivamente) nos produtores rurais que relataram já ter sofrido intoxicação por agrotóxicos. Dentre os principais sintomas estão dor de cabeça, irritação na pele e tontura, como pode ser verificado na Tabela 3. Dos trabalhadores que apresentaram sintomas, $78 \%$ utilizam agrotóxicos há cerca de 10 a 30 anos. Além disso, $65 \%$ afirmam aplicar esses produtos no mínimo 4 vezes por mês.

Vinte um (7\%) dos trabalhadores rurais relataram ter sofrido intoxicação por agrotóxico, diagnosticada por um profissional da saúde, pelo menos uma vez na vida. Esses produtores são em sua maioria homens (86\%), com idade entre 21 a 71 anos e média de 36,9 anos $( \pm 11,9)$. Quanto à exposição a agrotóxico, 19\% lidam com os produtos há menos de um ano e $76 \%$ de 10 a 30 anos. A aplicação na lavoura ocorre de 4 a mais vezes por mês para cerca de $65 \%$ desses trabalhadores. Na Tabela 4, que aponta as principais características sociodemográficas e do uso de agrotóxicos entre os produtores que se queixaram de sintomas ou de intoxicação, foi observado que a associação entre into- 
Tabela 2 - Características do uso de agrotóxicos no pólo fruticultor do Vale do São Francisco

Table 2 - Characteristics of pesticide use in fruit farming of the San Francisco Valley

\begin{tabular}{|c|c|c|}
\hline Características & $\mathbf{N}$ & Resultados (\%) \\
\hline \multirow[t]{3}{*}{ Classe dos agrotóxicos } & 27 & 47 Inseticidas \\
\hline & 22 & 40 Fungicidas \\
\hline & 7 & 13 Outros \\
\hline \multirow[t]{6}{*}{ Grupo Químico } & 11 & 19 Organofosforados \\
\hline & 8 & 14 Piretróides \\
\hline & 4 & 6 Benzimidazol \\
\hline & 4 & 6 Triazol \\
\hline & 3 & 5 Necotinoídes \\
\hline & 26 & 45 Outros \\
\hline \multirow[t]{4}{*}{ Classificação toxicológica } & 10 & 18 Extremamente tóxicos \\
\hline & 11 & 19 Altamente tóxicos \\
\hline & 22 & 40 Medianamente tóxicos \\
\hline & 13 & 23 Pouco tóxicos \\
\hline \multirow[t]{5}{*}{ Classificação ambiental } & 5 & 9 Altamente perigoso \\
\hline & 23 & 42 Muito perigoso \\
\hline & 20 & 35 Perigoso \\
\hline & 2 & 4 Pouco perigoso \\
\hline & 6 & 10 em classificação \\
\hline Orientação técnica para a compra de agrotóxicos & 130 & $\begin{array}{c}46 \text { não possuem ou buscam informações } \\
\text { nas lojas de produtos agrícolas }\end{array}$ \\
\hline $\begin{array}{l}\text { Utilização de receituário na compra de } \\
\text { agrotóxicos }\end{array}$ & 221 & 78 não utilizam \\
\hline \multirow[t]{4}{*}{ Tipo de aplicador dos agrotóxicos } & 125 & 44 trator \\
\hline & 102 & 36 pulverizador costal \\
\hline & 50 & 18 tração animal \\
\hline & 6 & 2 outros \\
\hline Leitura do rótulo & 74 & 26 não lêem \\
\hline \multirow[t]{2}{*}{ Uso de EPI } & 54 & 19 não utilizam \\
\hline & 25 & 9 utilizam de maneira incompleta \\
\hline
\end{tabular}

xicação e baixa escolaridade é corroborada pela variável leitura do rótulo, e também que há associação entre o uso incompleto ou o não uso de EPI e a intoxicação, assim como de quem aplica agrotóxico mais de 02 vezes por mês. Já ter ou não orientação técnica não apresenta diferença significativa na intoxicação por esses compostos.

\section{Discussão}

No presente trabalho foi observada predominância da população masculina entre os trabalhadores da fruticultura no submédio do vale do São Francisco, contrariando o que foi publicado por Branco e
Vainsencher ${ }^{13}$, que afirmam a preferência do empresariado dessa região em empregar mão-de-obra feminina por considerá-la mais criteriosa, delicada e produtiva. Entretanto, concordam com diversos trabalhos que apontam a população masculina como a mais empregada no meio rural ${ }^{1,14}$, inclusive na fruticultura ${ }^{15}$.

A condição de vida da maioria dos trabalhadores está comprometida pela baixa renda e pela sazonalidade dos contratos. Trata-se de importante vulnerabilidade, pois, em decorrência disso, há repercussão direta nas condições de moradia, nutrição, escolaridade etc. Em relação à baixa escolaridade há uma vulnerabilidade maior para 
Tabela 3 - Principais queixas de saúde dos produtores rurais no pólo fruticultor do Vale do São Francisco

Table 3 - Main health complaints in fruit farming workers of the San Francisco Valley

\begin{tabular}{lcc}
\hline Queixas & $\mathbf{N}^{\circ}$ de ocorrência & $\%$ \\
\hline Dor de cabeça & 53 & 73 \\
Irritação na pele & 19 & 26 \\
Tontura & 18 & 25 \\
Espirros & 15 & 20 \\
Coceira intensa & 10 & 14 \\
Fraqueza & 8 & 11 \\
Lacrimejamento & 8 & 11 \\
Vômito & 8 & 11 \\
Dificuldade respiratória & 5 & 7 \\
Visão turva ou embaçada & 4 & 5 \\
Confusão mental & 4 & 5 \\
Náuseas & 4 & 5 \\
Perda de apetite & 2 & 3 \\
Formigamento nas pálpebras e nos lábios & 2 & 3 \\
Mancha de pele & 2 & 3 \\
Febre muito alta & 1 & 1,4 \\
\hline
\end{tabular}

as situações de risco, pelo fato de implicar na dificuldade de leitura dos rótulos dos agrotóxicos. Ainda com relação a este tema, foi observado que a linguagem utilizada para informar as características do produto e os cuidados com o manuseio constitui barreira(s) à comunicação de risco para a saúde e para o ambiente ${ }^{16,17}$.

Entre os agrotóxicos, $37 \%$ são extremamente ou altamente tóxicos para o homem e $42 \%$ muito perigosos para o ambiente, incluindo o Folisuper, agrotóxico mais aplicado na região e utilizado indiscriminadamente, uma vez que, apesar de ser indicado pelo Ministério da Agricultura para as culturas de algodão, feijão, milho, soja e trigo, tem a orientação dos vendedores para as culturas de cebola, manga e tomate ${ }^{18}$.

A maioria dos trabalhadores não possui orientação técnica no local de trabalho, obtendo-a nas lojas de venda de produtos agrícolas. No entanto, $21 \%$ dos vendedores das lojas de região não estão devidamente preparados para orientar os agricultores ${ }^{18}$.

Apesar da metodologia criteriosa desse estudo, pode ter ocorrido casualidade reversa, viés de memória e de informação. Como pode ser observado no fato de o trabalhador ter ou não orientação técnica no manuseio do agrotóxico, isso parece não fazer diferença quanto à intoxicação, uma vez que a falta de orientação técnica, que ocorre em várias regiões do país ${ }^{14,17,19}$, expõe os trabalhadores a situações de risco, já que estes não utilizam nenhum critério de dosagem em relação ao tipo de cultura e ao tamanho da plantação, e acabam empregando produtos não específicos para determinadas doenças ${ }^{20}$.

Os organofosforados são alguns dos produtos mais utilizados na fruticultura da região. No Brasil, são os responsáveis pelo maior número de intoxicações agudas e mortes registradas. Esse grupo químico acumula acetilcolina nas sinapses nervosas, desencadeando uma série de efeitos parassimpaticomiméticos, podendo causar neurotoxicidades e neuropatias periféricas $\operatorname{tardias}^{21}$.

As queixas de saúde referidas foram as esperadas para grupos expostos aos agrotóxicos $^{22,23}$, como mostram Araújo et al. ${ }^{24} \mathrm{em}$ 
Tabela 4 - Características sociodemográficas e do uso de agrotóxicos entre os produtores que se queixaram de sintomas ou de intoxicação.

Table 4 - Socio-demographic characteristics and use of pesticides among workers who have complained of symptoms of intoxication.

\begin{tabular}{|c|c|c|c|}
\hline Características & $\begin{array}{l}\text { Trabalhadores que se } \\
\text { queixaram de sintomas ou } \\
\text { Intoxicação por agrotóxicos }\end{array}$ & $\begin{array}{l}\text { Trabalhadores não } \\
\text { intoxicados e sem } \\
\text { sintomas }\end{array}$ & RP (IC95\%) \\
\hline \multicolumn{4}{|l|}{ Escolaridade } \\
\hline Analfabeto & 15 & 60 & $1,83(1,18-2,84)$ \\
\hline $1^{\circ}, 2^{\circ}$ e $3^{\circ}$ grau completo & 19 & 189 & $p=0,023$ \\
\hline \multicolumn{4}{|l|}{ Aplicação de agrotóxico } \\
\hline Até 2 vezes por mês & 28 & 45 & $0,63(0,43-0,93)$ \\
\hline Mais de 3 vezes por mês & 46 & 143 & $p=0,035$ \\
\hline \multicolumn{4}{|l|}{ Orientação quanto ao uso de agrotóxico } \\
\hline Possui orientação técnica & 56 & 161 & $0,99(0,64-1,52)$ \\
\hline Não possui orientação técnica & 19 & 47 & $p=0,913$ \\
\hline $\begin{array}{l}\text { Possui orientação de agrônomos ou } \\
\text { técnicos agrícolas }\end{array}$ & 36 & 108 & $1,12(0,76-166)$ \\
\hline $\begin{array}{l}\text { Possui orientação do proprietário, } \\
\text { encarregado da fazenda, vendedor ou não } \\
\text { possui orientação }\end{array}$ & 39 & 100 & $p=0,654$ \\
\hline \multicolumn{4}{|l|}{ Leitura do rótulo } \\
\hline Sim & 48 & 163 & $1,72(1,18-2,51)$ \\
\hline Não & 29 & 45 & $p=0,01$ \\
\hline \multicolumn{4}{|l|}{ Uso do EPI } \\
\hline Usa EPI & 60 & 169 & $1,06(0,65-1,72)$ \\
\hline Não usa EPI & 15 & 39 & $p=0,948$ \\
\hline Usa EPI completo & 42 & 162 & $2,03(1,39-2,95)$ \\
\hline Não usa ou usa EPI incompleto & 33 & 46 & $p<0,001$ \\
\hline
\end{tabular}

um estudo com produtores de tomate no Vale do São Francisco que cita tontura e dor de cabeça como os principais incômodos relatados, e Castro e Confalonieri ${ }^{20}$, em estudo sobre o uso de agrotóxico em cachoeira de Macau. Essas queixas podem variar intensamente, dependendo das vulnerabilidades individuais e coletivas, sendo comumente diagnosticadas como intoxicação aguda as queixas imediatas pós-exposição aos agrotóxicos (fraqueza, vômitos, náuseas, convulsões, dores de cabeça). No entanto, as intoxicações crônicas necessitam de uma atenção médica ainda maior para o seu diagnóstico, o que envolveria um raciocínio clínico-epidemiológico mais atento, espe- cialmente envolvendo profissionais da rede básica de saúde e especialistas, tais como dermatologistas, neurologistas, imunologistas, cancerologistas, gastrenterologistas, nefrologistas e pediatras ${ }^{25}$.

Neste sentido, faz-se necessário, nos trabalhadores rurais do submédio do Vale do São Francisco, realizar estudos toxicológicos, clínicos e epidemiológicos analíticos para compreender melhor os impactos do uso de agrotóxicos na saúde desses trabalhadores, tendo em vista as limitações dos estudos realizados por morbidade referida. Para isso é fundamental que os médicos estejam treinados para o diagnóstico de intoxicações agudas e crônicas e que o sistema 
de informação toxicológico e epidemiológico esteja organizado e articulado em todas as cidades da região.

A prevalência de $7 \%$ de pelo menos uma intoxicação por agrotóxico no decorrer da vida dos agricultores, esta próxima das encontradas em regiões Brasileiras. Faria et al. ${ }^{15}$ descreveram a prevalência de $12 \%$ de intoxicação por agrotóxicos em trabalhadores rurais da Serra Gaúcha. Já Vander Hoek et al. ${ }^{26}$ encontraram uma variação de 7 a $22 \%$ em regiões do Sri Lanka. e London et al. ${ }^{27}$ de $9 \%$ na África do Sul.

A notificação de intoxicação por agrotóxicos está estabelecida no Sistema Nacional de Agravos Notificados - SINAN. No entanto, encontra-se incipiente na rede de saúde dos municípios da região. Segundo a Secretaria de Vigilância Sanitária, de 20012006 foram registrados apenas 59 casos de intoxicação por agrotóxicos em Petrolina, sendo em primeiro lugar por tentativa de suicídio e em segundo por acidentes de trabalho, contaminação acidental e ingestão de alimentos contaminados. A subnotificação, segundo Ramos et al. ${ }^{12}$ é causada pelos seguintes fatos: o agricultor com sinais de intoxicação não procura o serviço de saúde; falta de conhecimento das equipes de saúde sobre os sinais e sintomas de intoxicação crônica; confusão com outras patologias; falta de diagnostico e, acrescentamos, por falta de registro.

Outra vulnerabilidade observada no discurso dos trabalhadores entrevistados é o fato de, mesmo quando realizado o diagnóstico de intoxicação química (aguda ou crônica), os trabalhadores são mantidos, em sua maioria, na atividade ocupacional, não recebendo da previdência social o reconhecimento de portador de doença profissional e o amparo beneficiário devido. O efeito cumulativo da exposição crônica pode significar danos irreparáveis e incapacidade permanente para o trabalho, e este fato parece não encontrar suporte na ação da previdência social. Uma solução para minimizar essa questão é apontada por Mota eYoung ${ }^{28}$, que sugerem o restabelecimento dos impostos sobre agrotóxicos no Brasil ou a promoção de tributação que sirva como instrumento econômico para gestão ambiental. Segundo Sobreira e Adis$\mathrm{si}^{29}$, se os agrotóxicos são reconhecidamente substâncias que provocam lesões crônicas, os impostos fortaleceriam a previdência nacional no sentido de ampliar os benefícios concedidos aos trabalhadores lesionados, inválidos e familiares de trabalhadores mortos.

Há necessidade de um diagnóstico situacional da problemática ambiental, em especial relacionado à contaminação do solo pela quantidade de embalagens descartadas de maneira não segura, principalmente pela falta da coleta feita pela ACAVASF e também de contaminação hídrica, por conta do processo de irrigação.

Sem entrar na discussão da eficácia dos Equipamentos de Proteção Individual (EPI) de modo geral, o fato de nos locais de trabalho estes nem sempre estarem apropriados ou serem insuficientes para a manipulação de produtos químicos ${ }^{30}$, revela que os trabalhadores estão ainda mais vulneráveis às exposições nocivas dos agrotóxicos. Como foi observado, a pulverização dos agrotóxicos, seja por trator, costal ou por tração animal, é o meio empregado para aplicação dos produtos, acarretando exposição massiva e desconforto, posto que os EPIs disponibilizados para os trabalhadores não estão adequados às condições climáticas ${ }^{17,20}$. O submédio do Vale do São Francisco possui temperatura média de $28^{\circ} \mathrm{C}$. Como a exposição dérmica é apontada como a principal vias de absorção de agrotóxicos ${ }^{31-34}$, o agricultor que se encontra com todas as partes do corpo com proteção tende a estar mais protegido. Esse estudo apontou que os trabalhadores que não usavam o EPI ou o utilizavam de maneira incompleta sofreram mais intoxicação. Porém o fato de usar o EPI não interferiu na proteção esperada a intoxicação por agrotóxico.

Segundo Oliveira-Silva et $a l .^{35}$, os indicadores socioeconômicos levantam evidências acerca da complexidade do processo de exposição/intoxicação por agrotóxicos. A ausência de ações integradas que atuem 
na diminuição da desigualdade social, na melhoria da escolaridade e no preparo das equipes de saúde, é reveladora da vulnerabilidade institucional observada nesse contexto produtivo analisado.

A falta de uma política efetiva de fiscalização no acompanhamento técnico e no controle de agrotóxicos nessa importante região produtora de frutas do país, e integrada no mercado globalizado, revela que o parâmetro que interessa aos tomadores de decisão é apenas o da produção. A saúde e o ambiente estão longe de uma atenção adequada.

\section{Referências}

1. Moreira JC, Jacob SC, Peres F, Lima JS, Meyer A, OliveiraSilva JJ et al. Avaliação integrada do impacto do uso de agrotóxicos sobre a saúde humana em uma comunidade agrícola de Nova Friburgo, RJ. Ciênc Saúde Coletiva 2002; 7 (2): 299-311.

2. World Health Organization. Public health impact of pesticides used in agriculture. Geneva: World Health Organization;1995

3. Peres F, Moreira JC. É veneno ou é remédio? Agrotóxicos, saúde e ambiente. Rio de Janeiro: FIOCRUZ; 2003.

4. Rueg EF. Impacto dos agrotóxicos sobre o ambiente, a saúde e a sociedade. São Paulo: Ícone; 1991.

5. Augusto LGS, Gurgel IGD, Florêncio L, Araújo ACP. Exposição ocupacional aos agrotóxicos e riscos sócioambientais: subsídio para ações integradas no estado de Pernambuco. In: Augusto LGS, Florêncio L, Carneiro RM. Pesquisa (ação) em saúde ambiental - contexto, complexidade, compromisso social. Recife: Editora Universitária; 2005. p. 57-69.

6. Pavão AC, Leão, MBC. Riscos de carcinogênese química no controle do Aedes. In: Augusto LGS, Carneiro RM, Martins PH. Abordagem Ecossistêmica: Ensaios para o Controle da Dengue. Recife: Editora Universitária; 2005. p. 213-26.

7. Perez F, Oliveira-Silva JJ, Della-Rosa HV, De Luca SR. Desafios ao estudo da contaminação humana e ambiental por agrotóxicos. Ciênc Saúde Coletiva 2005; 10: 27-37.

8. Popper R, Andino K, Bustamante M, Hernandez B, Rodos L. Knowledge and beliefs regarding agricultural pesticides in rural Guatemala. Environ Manage 1996; 20 : 241-8.

9. Mwanthi AM, Mseh SB,, Kimani NV. Patterns of agrochemical handling and community Response in Central Kenya. J Environ Health 1993; 55 (7): 11-16.

10. McDougall L, Magloire L, Hospedales CJ, Tollefson JE, Ooms M, Singh NC et al. Attitudes and practices of pesticide uses in Saint Lucia, West Indies. Bulletins of PAHO 1993; 27: 43-51.
11. Stewart DJ. Pesticide use, habits and health awareness among Egyptian Farmers. AMBIO 1996; 25: 425-6.

12. Ramos LO, Vilas Boas JM, da Silva Junior CA, Bedor, CNG. "Queixas" Sintomáticas Associadas ao uso de agrotóxicos em trabalhadores rurais da hortifruticultura no Vale do São Francisco. Rev Ciênc Saúde Nova Esperança 2006; 4: 145-57.

13. Branco AM, Vainsencher AS. Gênero e Globalização no Vale do São Francisco. Fundação Joaquim Nabuco. 116/2001. Disponível em http://www.fundaj.gov.br/ tpd/116.html [Acessado em 20 de janeiro de 2008].

14. Soares W, Almeida RMVR, Moro S. Trabalho rural e fatores de risco associados ao regime de uso de agrotóxicos em minas Gerais, Brasil. Cad Saúde Pública 2003; 19: 1117-27.

15. Faria NMX, Facchini LA, Fassa, AG Tomasi E. Trabalho rural e intoxicações por agrotóxicos. Cad Saúde Pública 2004; 20: 1298-308.

16. Pinto JB. A educação de adultos e o desenvolvimento rural. In: Werthein J, Bordenave JD. Educação rural no terceiro mundo: experiências e novas alternativas. Rio de Janeiro: Paz e Terra; 1985. p. 161-98.

17. Recena MCP, Caldas ED. Percepção de risco, atitudes e práticas no uso de agrotóxicos entre agricultores de Culturama, MS. Rev Saúde Pública 2008; 42: 294-301.

18. Bedor CNG, Ramos LO, Rêgo MAV, Pavão AC, Augusto LGS. Avaliação e reflexão da comercialização e utilização de agrotóxicos na região do submédio do vale do São Francisco. Rev Baiana Saúde Pública 2007; 31: 68-76.

19. Peres F, Rozemberg B, Alves SR, Moreira JC, OliveiraSilva JJ. Comunicação relacionada ao uso de agrotóxicos em região agrícola do Estado do Rio de Janeiro. Rev Saúde Pública 2001; 35: 564-70

20. Castro JSM, Confaloniere U. Pesticide use in Cachoeiras de Macacu, Rio de Janeiro, Brazil. Ciênc. Saúde Coletiva 2005; 10: 473-82 
21. Caldas ED, de Souza LC. Assessment of the chronic risk for ingestion of pesticide residues in the Brazilian diet. Rev Saúde Pública 2000; 34: 529-37.

22. Fehlberg LCC, Lutz LV, Moreira AH. Agrotóxicos e seus efeitos sócio-culturais: Zona rural do Valão São Lourenço, Santa Tereza, ES, Brasil. Natureza on line 2003; 1:51-55. Disponível em http://www.naturezaonline. com.br [Acessado em 5 de janeiro de 2008].

23. Araújo AJ, Lima JS, Moreira JC, Jacob SC, Soares MO, Monteiro MC et al. Exposição múltipla a agrotóxicos e efeitos à saúde: estudo transversal em amostra de 102 trabalhadores rurais, Nova Friburgo, RJ. Ciênc Saúde Coletiva 2007; 12: 115-130.

24. Araújo ACP, Nogueira DP, Augusto LGS. Pesticide impact on health: a study of tomato cultivation. Rev Saúde Publica 2000; 34: 309-13

25. Wilson JS, Otsuki T. To spray or not to spray: pesticides, banana exports, and food safety. Food Policy 2004; 29: 131-46.

26. Van der Hoek W, Konradsen F, Athukorala K, Wanigadewa T. Pesticide poisoning: a major health problem in Sri Lanka. Soc Sci Med 1998; 46: 495-504.

27. London L, Ehrlich RI, Rafudien S, Krige F, Vurgarellis P. Notification of poisoning in the Western Cape, 1987 1991. S Afr Med J 1994; 84: 269-72.

28. Mota RS, Young CEF. Instrumentos econômicos para a gestão ambiental no Brasil. 1997. Disponível em http:// www.mma.gov.br [Acessado em 5 de janeiro de 2008].

29. Sobreira AEG, Adissi PJ. Agrotóxicos: falsas premissas e debates. Ciênc Saúde Coletiva 2003; 8: 985-90
30. Chester MH, Adam AV, Inkmann-Koch A, Litchfiel MH, Sabapathy R, Tuiman CP. Field evaluation of protective equipment for pesticide appliers in a tropical climate. In: Forget G, Goodman T, Villiers A. Impact of pesticide use on health in developing countries. Proceedings of a symposium held in Ottawa, Canada: International Development Research Centre; 1993. p. 116-123

31. Levine RS, Doull J. Global estimates of acute pesticide morbidity and mortality. Rev Environ Contam Toxicol 1992; 129: 29-50.

32. Kishi M, Hirschhorn N, Djajadisastra M, Satterlee LN, Strowman S, Dilts R. Relationship of pesticide spraying to signs and symptoms in Indonesian farmers. Scand $J$ Work Environ Health 1995; 21: 124-33.

33. Machado Neto JG. Segurança no trabalho com agrotóxicos em cultura de eucalipto. São Paulo: Fundação de Estudos e Pesquisas em Agronomia, Medicina Veterinária e Zootecnia; 2001.

34. Bellini TM, Oliveira ML, Machado Neto JG. Eficiência de vestimentas hidrorrepelentes novas na proteção do tratorista em pulverizações de agrotóxicos em goiaba com o urbopulverizador. Rev Bras Frutic 2008; 30: 106111.

35. Oliveira-Silva JJ, Alves SR, Meyer A, Perez F, Sarcinelli PN, da Costa Mattos RC et al. Influence of socioeconomic factors on the pesticides poisoning, Brazil. Rev Saúde Publica 2001; 35: 130-135

Recebido em: 05/12/07

Versão final reapresentada em: 21/11/08 Aprovado em: 18/12/08 